\title{
Parallel Flow of a Pressure-Dependent Viscosity Fluid through Composite Porous Layers
}

\author{
M. S. ABU ZAYTOON ${ }^{1}$ \\ M. H. HAMDAN ${ }^{2}$ \\ Department of Mathematics and Statistics \\ University of New Brunswick \\ 100 Tucker Park Road, Saint John, N.B., \\ CANADA
}

\begin{abstract}
Flow of a fluid with pressure-dependent viscosity through a composite of two porous layers is considered in this work in an attempt to validate velocity and shear stress continuity conditions at the interface, and are popular in the study of flow over porous layers and through composite layers when viscosity of the fluid is constant. For the current problem, conditions at the interface between the porous layers reflect continuity assumptions of velocity and shear stress, with additional continuity assumptions on pressure and viscosity. Viscosity is assumed to vary continuously and exponentially across the layers as a function of pressure. Analytical solutions are obtained to illustrate the effects of flow and media parameters (Darcy numbers, layer thicknesses, angle of inclination, and viscosity adjustment parameter) on the dynamic behaviour of pressure-dependent viscosity fluids in porous structures. All computations, simulations and graphs in this work have been carried out and obtained using Maple 2020 software package.
\end{abstract}

Key-Words: - Composite porous layers; Pressure-Dependent Viscosity; Inclined Plane.

Received: April 8, 2021. Revised: January 5, 2022. Accepted: January 18, 2022. Published: March 1, 2022.

\section{Introduction}

In this work, we initiate the study of flow of fluids with pressure-dependent viscosities through composite porous layers. Flow of fluids with constant viscosities through layered porous media have been extensively studied ( $c f .[1,2,3]$ and the references therein). Flow of fluids with pressure-dependent viscosities through free-space has also received considerable attention in the literature, ( $c f .[4,5,6,7$, $8]$ and the references therein). However, only recently models of flow of fluids with pressuredependent viscosities through porous media have been derived $[9,10,11,12]$.

Fluid viscosity variations include changes in viscosity due to temperature, pressure and shearthinning. Many fluids, such as paint and polymers exhibit behaviours in which a fluid becomes either thicker, or thinner when sheared, [13, 14, 15, 16, 17]. Nakshatrala \& Rajagopal [17] provided an account of these variations. Studies of fluid viscosity variations and viscosity dependence on pressure can be traced to back to the nineteenth century and the work of various authors, including Stokes [18], Barus [19, 20]. More recently, experimental studies confirm dependence of viscosity on pressure, $[7,8,21]$.

Barus $[19,20]$ suggested two relationships for dependence of viscosity on pressure, an exponential and a linear relationship. The Barus relationships received considerable analysis in the literatrs, (cf. [22] and the references therein). A model describing the dependence of viscosity on pressure, temperature and density has been reported in Szeri [5]. Other models of dependence have been discussed in the literature, $[23,24]$.

Interest in fluids with pressure-dependent viscosities in porous media stems from their industrial applications in the oil industry (enhanced oil recovery and geological carbon sequestration), food and polymer processing, in the pharmaceutical industry, in thin film lubrication and in filtration 
problems [6, 15, 17, 22, 25, 26]. Fusi et.al. [25] provided elegant modelling of three important filtration problems involving pressure-dependent viscosity fluids in porous media, and Housiadas et.al. [26] initiated studies of compressible fluids with pressure-dependent viscosity, in their development of new analytic solutions of weakly compressible Newtonian Poiseuille flows. These and many other applications initiated the recent interest in the modelling and simulation of flow through porous media with a focus on fluids with variable or stratified viscosities, and fluids with pressuredependent viscosities, [12, 11, 12, 17].

Models describing the flow of fluids with pressuredependent viscosity through porous media have been derived, discussed or otherwise analyzed by various authors, including Srinivasan and Rajagopal [10], Nakshatrala and Rajagopal [17], Kannan and Rajagopal [13], Abu Zaytoon et.al. [11], Alharbi, et.al. [12], Of interest to the current work is the Brinkman-type model, [10, 13, 17], in which the flow through a rigid porous structure is described by the following equations of continuity and momentum, respectively, written here for steady flow:

$\nabla \cdot \vec{u}=0$

$\rho \vec{u} \cdot \nabla \vec{u}=-\nabla p+\nabla \cdot \vec{T}-\lambda(p) \vec{u}+\rho \vec{G}$

$\vec{T}=-p \vec{I}+2 \mu(p) \vec{A}$

is the Cauchy stress tensor in which

$\vec{A}=\frac{1}{2}\left(\nabla \vec{u}+(\nabla \vec{u})^{T}\right.$

where $\vec{u}$ is the velocity vector field, $p$ is the pressure, $\rho$ is the fluid density, $\rho \vec{G}=$ $\left(\rho G_{1}, \rho G_{2}, \rho G_{3}\right)$ is the body force, $\mu=\mu(p)$ is the variable viscosity, and $\lambda(p)$ is a drag function that has been given various forms as discussed by Kannan and Rajagopal [13], and include exponential and polynomial forms in terms of pressure. Governing equations for flow in the channel are the equation of continuity and the Navier-Stokes equations with pressure-dependent viscosity. These equations are similar in form to equations (1) and (2) with $\lambda(p)=$ 0 in (2a).

When the drag function is expressed as the ratio between viscosity of the fluid and permeability of the porous medium, namely, $\lambda(p)=\mu / k$, equation (2) reduces to a model derived using intrinsic volume averaging, [11, 12]. This form of drag function facilitates studies of flow through constant and variable permeability porous media $[27,28]$. We implement this form in the current study where we consider the parallel flow of a fluid with pressuredependent viscosity through two porous layers inclined to the horizontal at an angle.

The flow domain of a porous channel inclined to the horizontal has been a model configuration for many problems, including thin film lubrication and wave, [29], and, we believe, it facilitates the introduction of a continuous pressure function on which viscosity depends. Conditions at the interface between the porous layer that emphasize continuity of pressure, viscosity, shear stress and velocity.

\section{Problem Formulation}

The steady flow of an incompressible fluid with pressure-dependent viscosity through a porous medium is governed by continuity and momentum equations (1) and (2), above, in which we take $\lambda(p)=\mu / k$.

Now, consider the flow through the configuration shown in Fig.1, where a fluid flows through two composite porous layers each with different permeability. We set layer 1 to $0 \leq y \leq h_{1}$ and layer 2 to $h_{1} \leq y \leq h$. Layers 1 and 2 are assumed to be parallel and meet at a sharp interface $y=h_{1}$ with angle of inclination $\vartheta$. The layers are bounded by solid, impermeable walls at $y=0$ and $y=h$.

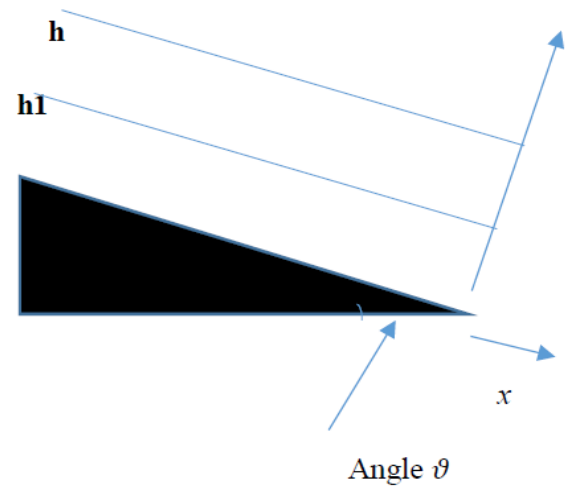

Fig. 1. Representative sketch

For the unidirectional flow at hand, the flow is in the $\mathrm{x}$-direction, taken along the inclined wall, with $\vec{u}=(u, 0)$. Continuity equation (1) implies that $u_{x}=$ 0 or $u=u(y)$. Equation (2) reduces to $-p_{y}+$ $\rho G_{2}=0$, where $\rho G_{2}$ is the body force component in the y-direction, namely, $-\rho g \cos \vartheta$. Flow in the $\mathrm{x}$ direction is under the effect of body force (gravity) whose component in the x-direction is $\rho g \sin \vartheta$. 
Pressure gradient in the $x$-direction is zero, $p_{x}=0$, which emphasizes that $p=p(y)$.

We now define the following dimensionless quantities and Darcy numbers with respect to total thickness of the flow domain and with respect to a characteristic velocity, $U$ :

$y^{*}=\frac{y}{h} ; u_{i}^{*}=\frac{u_{i}}{U} \varepsilon=\frac{h_{1}}{h} ; D a_{i}=\frac{k_{i}}{h^{2}}$ for $i=1,2 ;$

Dropping the asterisk $(*)$, the governing equations for the $i$ th layer, where $i=1,2$ refer to the lower and upper layer, respectively, take the following forms:

$\mu_{i} \frac{d^{2} u_{i}}{d y^{2}}+\frac{d \mu_{i}}{d y} \frac{d u_{i}}{d y}+\frac{\rho g h^{2} \sin \vartheta}{U}-\frac{\mu_{i}}{D a_{i}} u_{i}=0$

$\frac{d p_{i}}{d y}=-\rho g h \cos \vartheta$

At the solid boundaries, $y=0$ and $y=1$, the velocity vanishes. We also assume that the value of pressure at $y=1$ is given as $p_{0}$. Boundary conditions are thus as follows:

$u_{1}(0)=0$

$u_{2}(1)=0$

$p_{2}(1)=p_{0}$

$u_{1}(\varepsilon)=u_{2}(\varepsilon)$

$\mu_{1} \frac{d u_{1}}{d y}(\varepsilon)=\mu_{2} \frac{d u_{2}}{d y}(\varepsilon)$

$\mu_{1}(\varepsilon)=\mu_{2}(\varepsilon)$

$p_{1}(\varepsilon)=p_{2}(\varepsilon)$

$0 \leq y \leq \varepsilon$

$p_{2}(y)=p_{0}+(1-y) \rho g h \cos \vartheta ;$

$\varepsilon \leq y \leq 1$

At the interface between the two layers, the pressure is given by

$p_{1}(\varepsilon)=p_{2}(\varepsilon)=p_{0}+(1-\varepsilon) \rho g h \cos \vartheta$
In order to solve (16), we first need to specify viscosities $\mu_{i}$ as functions of pressure distributions (13) and (14). In this work we assume that viscosities vary exponentially according to Barus' relationship of the form:

$\mu_{i}\left(p_{i}\right)=A e^{\alpha p_{i}}$

where $A$ and $\alpha$ are positive constants, referred to as viscosity control parameters. Their role is to keep values of viscosities within realistic limits. We note that $\mu_{1}\left(p_{1}\right)$ and $\mu_{2}\left(p_{2}\right)$ are equal at the interface, $y=$ $\varepsilon$, hence condition (11) is satisfied. Equations (4) thus take the following form:

$\frac{d^{2} \bar{u}_{i}}{d \bar{y}^{2}}-\beta \frac{d \bar{u}_{i}}{d \bar{y}}-\frac{\bar{u}_{i}}{D a_{i}}=-\gamma e^{\beta \bar{y}}$

where

$\beta=\alpha \rho g h \cos \vartheta$

$\gamma=\frac{\rho g h^{2} \sin \vartheta}{U A} e^{-\beta-\alpha p_{0}}$

\section{Problem Solution}

\subsection{Velocity Profiles}

General solutions to equations (17) are given by:

$u_{1}=c_{1} e^{m_{1} y}+c_{2} e^{m_{2} y}+\gamma D a_{1} e^{\beta y} ;$

$0<y \leq \varepsilon$

$u_{2}=d_{1} e^{r_{1} y}+d_{2} e^{r_{2} y}+\gamma D a_{2} e^{\beta y} ;$

$\varepsilon \leq y<1$

where $c_{1}, c_{2}, d_{1}$ and $d_{2}$ are arbitrary constants and

$m_{1}=\frac{\beta+\sqrt{\beta^{2}+\frac{4}{D a_{1}}}}{2}, \quad m_{2}=\frac{\beta-\sqrt{\beta^{2}+\frac{4}{D a_{1}}}}{2}, \quad r_{1}=$
$\frac{\beta+\sqrt{\beta^{2}+\frac{4}{D a_{2}}}}{2}, \quad r_{2}=\frac{\beta-\sqrt{\beta^{2}+\frac{4}{D a_{2}}}}{2}$

Velocity at the interface is given by either (20) or (21), evaluated at $y=\varepsilon$, namely 
$u_{1}(\varepsilon)=c_{1} e^{m_{1} \varepsilon}+c_{2} e^{m_{2} \varepsilon}+\gamma D a_{1} e^{\beta \varepsilon}$

$u_{2}(\varepsilon)=d_{1} e^{r_{1} \varepsilon}+d_{2} e^{r_{2} \varepsilon}+\gamma D a_{2} e^{\beta \varepsilon}$.

Now, to find the values of arbitrary constants $c_{1}, c_{2}, d_{1}$ and $d_{2}$ appearing in (20) and (21), we apply equation (6), (7), (9) and (10) to obtain the following system of linear equations:

$c_{1}+c_{2}+\gamma D a_{1}=0$

$d_{1} e^{r_{1}}+d_{2} e^{r_{2}}+\gamma D a_{2} e^{\beta}=0$

$c_{1} e^{m_{1} \varepsilon}+c_{2} e^{m_{2} \varepsilon}=d_{1} e^{r_{1} \varepsilon}+d_{2} e^{r_{2} \varepsilon}+$

$\gamma e^{\beta \varepsilon}\left(D a_{2}-D a_{1}\right)$

$c_{1} m_{1} e^{m_{1} \varepsilon}+c_{2} m_{2} e^{m_{2} \varepsilon}=d_{1} r_{1} e^{r_{1} \varepsilon}+d_{2} r_{2} e^{r_{2} \varepsilon}+$

$\gamma \beta e^{\beta \varepsilon}\left(D a_{2}-D a_{1}\right)$

Equations (25)-(28) are written in the matrix form $A \vec{X}=\vec{B}$, which can be solved numerically using Maple, where

$$
\begin{gathered}
A=\left(\begin{array}{cccc}
1 & 1 & 0 & 0 \\
0 & 0 & e^{r_{1}} & e^{r_{2}} \\
e^{m_{1} \varepsilon} & e^{m_{2} \varepsilon} & -e^{r_{1} \varepsilon} & -e^{r_{2} \varepsilon} \\
m_{1} e^{m_{1} \varepsilon} & m_{2} e^{m_{2} \varepsilon} & -r_{1} e^{r_{1} \varepsilon} & -r_{2} e^{r_{2} \varepsilon}
\end{array}\right), \\
\vec{X}=\left(\begin{array}{c}
c_{1} \\
c_{2} \\
d_{1} \\
d_{2}
\end{array}\right), \vec{B}=\left(\begin{array}{c}
-\gamma D a_{1} \\
-\gamma D a_{2} e^{\beta} \\
\gamma e^{\beta \varepsilon}\left(D a_{1}-D a_{2}\right) \\
\gamma \beta e^{\beta \varepsilon}\left(D a_{1}-D a_{2}\right)
\end{array}\right)
\end{gathered}
$$

\subsection{Vorticity and Shear Stress}

Vorticities, $\omega_{1}$ in the lower layer and $\omega_{2}$ in the upper layer, take the following forms with the help of (20) and (21), respectively:

$\omega_{1}=-\frac{d u_{1}}{d y}=-\left[c_{1} m_{1} e^{m_{1} y}+c_{2} m_{2} e^{m_{2} y}+\right.$

$\left.\gamma \beta D a_{i} e^{\beta y}\right]$

$\omega_{2}=-\frac{d u_{2}}{d y}=-\left[d_{1} r_{1} e^{r_{1} y}+d_{2} r_{2} e^{r_{2} y}+\right.$

$\left.\gamma \beta D a_{2} e^{\beta y}\right]$

with values at the interface given by

$$
\omega_{1}(\varepsilon)=-\left[c_{1} m_{1} e^{m_{1} \varepsilon}+c_{2} m_{2} e^{m_{2} \varepsilon}+\gamma \beta D a_{1} e^{\beta \varepsilon}\right]
$$

$\omega_{2}(\varepsilon)=-\left[d_{1} r_{1} e^{r_{1} \varepsilon}+d_{2} r_{2} e^{r_{2} \varepsilon}+\gamma \beta D a_{2} e^{\beta \varepsilon}\right]$.

We note that $\omega_{1}(\varepsilon)=\omega_{2}(\varepsilon)$ as a consequence of conditions (10) and (11).

The shear stresses, $\tau_{1}$ in the lower layer $\tau_{2}$ in the upper layer, are written as follows with the help of (20) and (21), respectively:

$\tau_{1}=\mu_{1} \frac{d u_{1}}{d y}=\mu_{1}\left[c_{1} m_{1} e^{m_{1} y}+c_{2} m_{2} e^{m_{2} y}+\right.$

$\left.\gamma \beta D a_{1} e^{\beta y}\right]$

$\tau_{2}=\mu_{2} \frac{d u_{2}}{d u}=\mu_{2}\left[d_{1} r_{1} e^{r_{1} y}+d_{2} r_{2} e^{r_{2} y}+\right.$

$\left.\gamma \beta D a_{2} e^{\beta y}\right]$

The shear stress at the interface $(y=\varepsilon)$ is given by:

$\tau_{1}(\varepsilon)=\mu_{1}(\varepsilon)\left[c_{1} m_{1} e^{m_{1} \varepsilon}+c_{2} m_{2} e^{m_{2} \varepsilon}+\right.$ $\left.\gamma \beta D a_{1} e^{\beta \varepsilon}\right]$

$\tau_{2}(\varepsilon)=\mu_{2}(\varepsilon)\left[d_{1} r_{1} e^{r_{1} \varepsilon}+d_{2} r_{2} e^{r_{2} \varepsilon}+\gamma \beta D a_{2} e^{\beta \varepsilon}\right]$

We note that $\tau_{1}(\varepsilon)=\tau_{2}(\varepsilon)$ due to $(10)$.

\section{Results and Discussion}

Results have been obtained for the following ranges that are representative of the media and flow parameters:

Ranges of Darcy Numbers: $D a_{1}=$ $1,0.1,0.01,0.001$ and 0.00001 , and $D a_{2}=$ 1 and 0.01 . We point out that when $D a_{1}=D a_{2}$ then the two layers behave like one layer.

Range of inclination angle $\vartheta$ : 30, 60, and 75 degrees. Range of $p_{0}: 2,3$, and 5 .

Range of $\varepsilon: 0.25,0.5$ and 0.75 .

Range of $\alpha: 0.01,0.1$ and 1 .

We can take $\rho g=1$ and $h=1$ as is Kannan and Rajagopal [13]. We also take $A=U=1$ as representative values.

\subsection{Velocity Profiles}

Velocities in the porous layers are given by equations (20) and (21), respectively. Effects of the various 
flow and medium parameters on the velocity profiles are discussed in what follows.

\subsubsection{Effects of Darcy Numbers}

Fig. 2 illustrates the effects of Darcy numbers on the velocity profiles across the two layers, when they are of the same thickness. As is well known, velocity increases with increasing Darcy number. When $D a_{1}=D a_{2}$, permeabilities are the same and the two layers behave like a single layer. The velocity profile shown for the case of $D a_{1}=D a_{2}=0.1$ is parabolic and symmetric about $y=0.5$.

In the lower layer, $y<0.5$, Fig. 2 shows the increase in velocity with increasing $D a_{1}$. By keeping $D a_{2}$ fixed at 0.1, Fig. 2 shows a greater influence on the upper layer by the lower layer with increasing $D a_{1}$. This influence results in distorting the parabolic shape of the velocity profile in the upper layer.

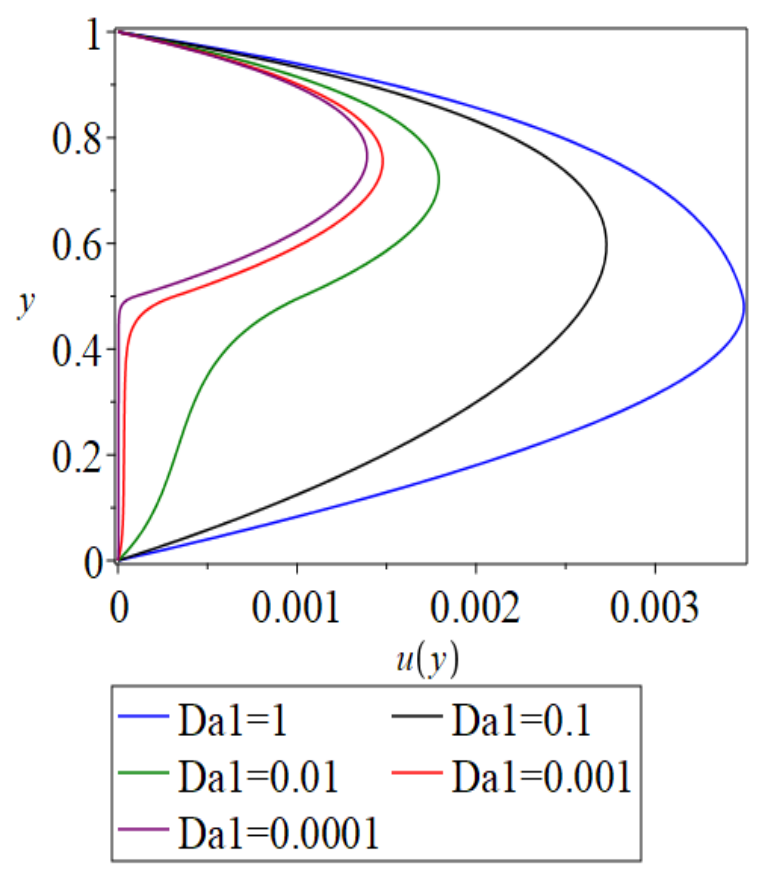

Fig. 2 Velocity across the two layers for various values of $D a_{1}$.

$\varepsilon=0.5, \alpha=1, U A=1, p_{0}=2, \vartheta=30, D a_{2}=$ 0.1 .

\subsubsection{Effects of $\varepsilon$}

The value of $\varepsilon$ represents the fraction of the total thickness of the flow domain that the lower layer occupies. When $\varepsilon=0.5$, both layers are of the same thickness.
In Fig. 3, we illustrate the effects of $\varepsilon$ when the lower layer has a higher Darcy number than the upper layer. This translates into the lower layer having a higher influence on the upper layer by virtue of the higher velocity associated with higher Da. With increasing $\varepsilon$, velocity at the interface increases, as per equations (23) and (24).

\subsubsection{Effects of $p_{0}$}

Fig. 4 illustrates the effects of $p_{0}$ on the velocity profile. It shows that with decreasing $p_{0}$ velocity increases due to the decrease in viscosity that is associated with decreasing $p_{0}$. Decreasing viscosity translates into a lesser resistance to the flow, hence a greater velocity.

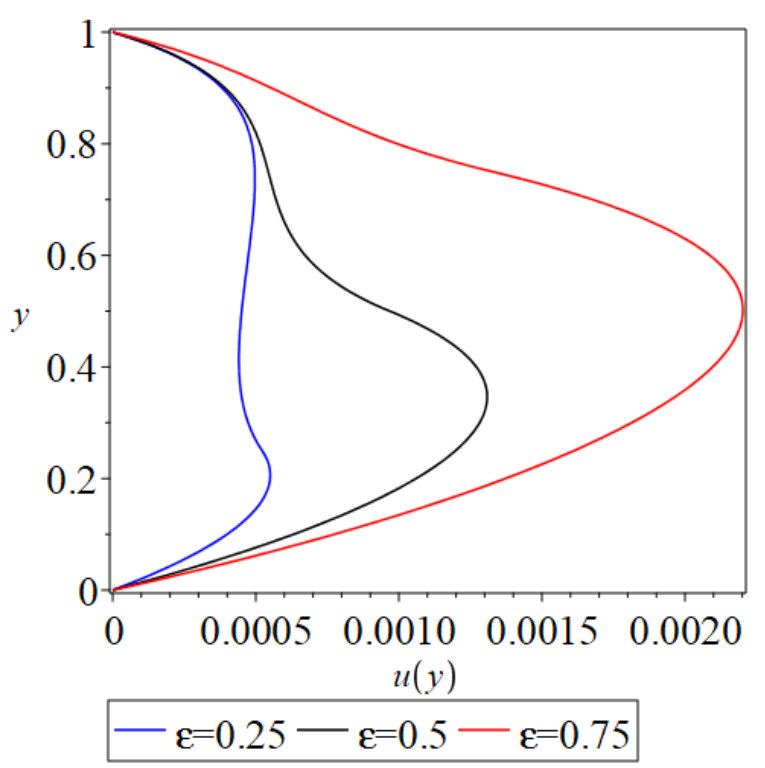

Fig. 3 Velocity across the two layers various values of $\varepsilon$.

$$
\begin{gathered}
\alpha=1 p_{0}=2, A U=1, \vartheta=30, D a_{2}=0.01, \text { and } \\
D a_{1}=0.1 .
\end{gathered}
$$

\subsubsection{Effects of $\vartheta$}

Associated with increasing angle of inclination is a decrease in the pressure distribution, hence a decrease in viscosity, hence an increase in velocity. In addition, with increasing inclination angle there is a greater gravitational force driving the flow. The net effect across the configuration is a velocity increases with increasing angle of inclination, as shown in Fig. 5. 


\subsubsection{Effects of $\alpha$}

For a given pressure distribution, when $\alpha$ increases then viscosity increases, which results in a decrease in velocity across the layers. This is illustrated in Fig. 6 which shows the slowing down of the flow as $\alpha$ increases.

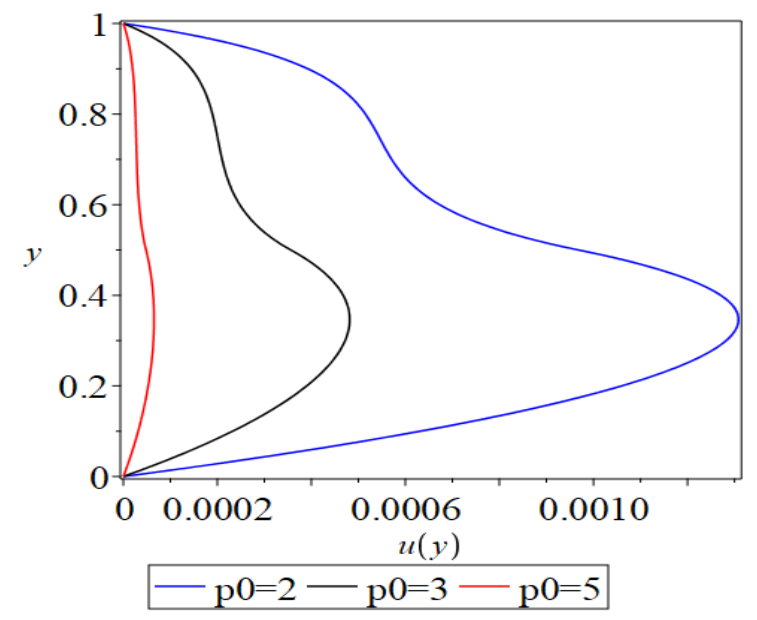

Fig. 4 Velocity across the two layers for various values of $p_{0}$.

$\varepsilon=0.5, \alpha=1, A U=1, \vartheta=30, D a_{2}=0.01$, and $D a_{1}=0.1$.

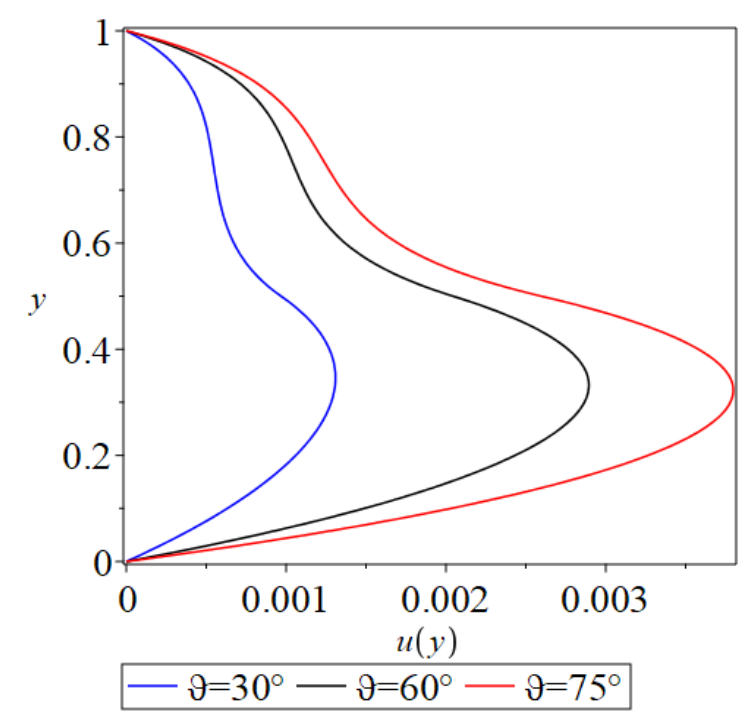

Figure 5 Velocity across the two layers for various values of $\vartheta$.

$\varepsilon=0.5, \alpha=1, A U=1, p_{0}=2, D a_{2}=0.01$, and $D a_{1}=0.1$.

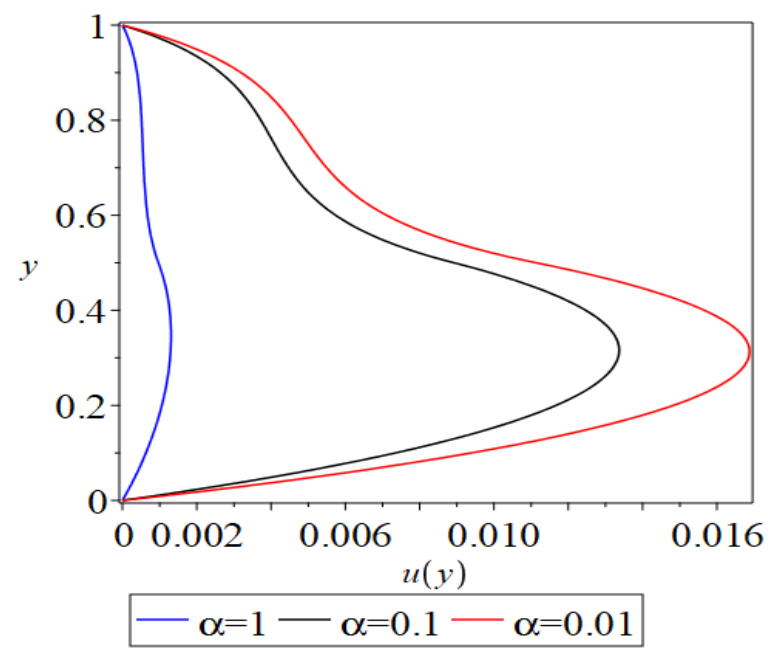

Fig. 6 Velocity across the two layers for various values of $\alpha$.

$\varepsilon=0.5, p_{0}=2, A U=1, \vartheta=30, D a_{2}=0.01$, and $D a_{1}=0.1$.

\subsection{Velocity, Vorticity and Shear Stress at the Interface}

Velocity, vorticity and shear stress at the interface $y=\varepsilon$ are tabulated, Tables $\mathbf{1}$ through $\mathbf{5}$, below for different parameters using expressions (23), (24), (32), (33), (36) and (37).

Table 1 illustrates the effects of Darcy number on interfacial quantities and shows the expected increase of velocity at the interface with increasing $D a_{1}$ for a fixed value of $D a_{2}$. This behaviour is in agreement with the effects of Darcy number $D a_{1}$ on the velocity profile, shown in Fig. 2. Vorticity decreases, numerically, with decreasing $D a_{1}$, and shear stress at the interface increases, numerically with decreasing $D a_{1}$ This is also in line with the velocity profiles depicted in Fig. 2 if one considers the behaviour of slopes of the velocity curves at the interface $y=\varepsilon$.

Also in line with the velocity profile figures, Fig. 2 to Fig. 6, are the effects of the parameters $\varepsilon, \alpha, \vartheta$ and $p_{0}$ on the velocity at the interface, illustrated in Tables 2 to $\mathbf{5}$ in which we document the values of vorticity and shear stress at the interface. We summarize their effects as follows. Increasing $\alpha$ results in a decrease in the interfacial velocity and a decrease in the vorticity, and a numerical increase in shear stress, as shown in Table 2.

Increasing $\varepsilon$, results in an increase in the interfacial velocity, and a corresponding increase in 
the vorticity, and a numerical decrease in the shear stress at the interface, as shown in Table 3.

Increasing $p_{0}$ results in a decrease in the interfacial velocity, and a decrease in the vorticity. The shear stress at the interface remains unaffected by changes in $p_{0}$, as shown in Table 4.

Increasing the angle of inclination, $\vartheta$, results in an increase in the interfacial velocity, as per Fig. 5, and a corresponding increase in the vorticity, while the shear stress at the interface decreases with increasing inclination angle, as shown in Table 5.

Table 1 Velocity at the interface for various $D a_{1} . \varepsilon=$ $0.5, \alpha=1, U A=1, p_{0}=2, \vartheta=30, D a_{2}=0.1$

\begin{tabular}{|l|l|l|l|}
\hline$D a_{1}$ & $u_{1}(\varepsilon)=u_{2}(\varepsilon)$ & $\omega_{1}(\varepsilon)=\omega_{2}(\varepsilon)$ & $\tau_{1}(\varepsilon)=\tau_{2}(\varepsilon)$ \\
\hline 1 & 0.0034873 & 0.00976403 & -0.08958714 \\
\hline 0.1 & 0.0026393 & 0.00401309 & -0.03682098 \\
\hline 0.01 & 0.0009683 & -0.0031813 & 0.0292059 \\
\hline 0.001 & 0.000283 & -0.0056960 & 0.0522625 \\
\hline 0.0001 & 0.000086 & -0.0064038 & 0.0587570 \\
\hline
\end{tabular}

Table 2 Velocity, Vorticity and Shear stress at the interface for different $\alpha . p_{0}=2, A U=1, \vartheta=30$, $D a_{2}=0.01$, and $D a_{1}=0.1, \varepsilon=0.5$

\begin{tabular}{|l|l|l|l|}
\hline$\alpha$ & $u_{1}(\varepsilon)=u_{2}(\varepsilon)$ & $\omega_{1}(\varepsilon)=\omega_{2}(\varepsilon)$ & $\tau_{1}(\varepsilon)=\tau_{2}(\varepsilon)$ \\
\hline 1 & 0.00096826 & 0.00476337 & -0.05426985 \\
\hline 0.1 & 0.00892301 & 0.05001707 & -0.06379441 \\
\hline 0.01 & 0.01113969 & 0.06319418 & -0.06475056 \\
\hline
\end{tabular}

Table 3 Velocity, Vorticity and Shear stress at the interface for different $\varepsilon$.

$\alpha=1 p_{0}=2, A U=1, \vartheta=30, D a_{2}=0.01$, and $D a_{1}=0.1$.

\begin{tabular}{|l|l|l|l|}
\hline$\varepsilon$ & $u_{1}(\varepsilon)=u_{2}(\varepsilon)$ & $\omega_{1}(\varepsilon)=\omega_{2}(\varepsilon)$ & $\tau_{1}(\varepsilon)=\tau_{2}(\varepsilon)$ \\
\hline 0.25 & 0.00052199 & 0.00131358 & -0.01858354 \\
\hline 0.5 & 0.00096826 & 0.00476337 & -0.05426985 \\
\hline 0.75 & 0.00132741 & 0.00812923 & -0.07458747 \\
\hline
\end{tabular}

Table 4 Velocity, Vorticity and Shear stress at the interface for different $p_{0}$.

$\varepsilon=0.5, \alpha=1, A U=1, \vartheta=30, D a_{2}=0.01$, and

\begin{tabular}{|l|l|l|l|}
\multicolumn{5}{|c|}{$D a_{1}=0.1}$. \\
\hline$p_{0}$ & $u_{1}(\varepsilon)=u_{2}(\varepsilon)$ & $\omega_{1}(\varepsilon)=\omega_{2}(\varepsilon)$ & $\tau_{1}(\varepsilon)=\tau_{2}(\varepsilon)$ \\
\hline 2 & 0.00096826 & 0.00476337 & -0.05426985 \\
\hline 3 & 0.00035620 & 0.00175235 & -0.05426985 \\
\hline 5 & 0.00004821 & 0.00023715 & -0.05426985 \\
\hline
\end{tabular}

Table 5 Velocity, Vorticity and Shear stress at the interface for different $\vartheta . \varepsilon=0.5, \alpha=1, A U=1$,

$$
p_{0}=2, D a_{2}=0.01 \text {, and } D a_{1}=0.1 \text {. }
$$

\begin{tabular}{|l|l|l|l|}
\hline$\vartheta$ & $u_{1}(\varepsilon)=u_{2}(\varepsilon)$ & $\omega_{1}(\varepsilon)=\omega_{2}(\varepsilon)$ & $\tau_{1}(\varepsilon)=\tau_{2}(\varepsilon)$ \\
\hline 30 & 0.00096826 & 0.00476337 & -0.05426985 \\
\hline 60 & 0.00204452 & 0.01072180 & -0.10172564 \\
\hline 75 & 0.00259720 & 0.01416932 & -0.11916257 \\
\hline
\end{tabular}

\section{Conclusion}

In this work, we modelled, and provided a solution to, parallel flow of a fluid with pressure-dependent viscosity through a composite of two inclined porous 
layers. It was assumed that permeabilities are constant and the flow in each layer is governed by Brinkman's equation for flow of fluids with variable viscosity. The layers were saturated by the same fluid. Pressure variations through the flow domain were described by a continuous linear function. Viscosity was then expressed as a continuous, exponential function of pressure. These choices of viscosity and pressure distributions helped maintain the continuity conditions at the interface between layers. Model equations were solved subject to continuity of velocity, pressure, viscosity and shear stress at the interface, and the no-slip condition on solid boundaries. Results obtained support the following conclusions:

1) Parameters that influence the flow are the angle of inclination, $\vartheta$, pressure condition $p_{0}$ at the upper channel wall, thickness $\varepsilon$ of the lower porous layer, viscosity adjustment parameter, $\alpha$, and the Darcy number of each layer.

2) For a given pressure distribution, viscosity distribution is the most sensitive parameter as it controls the behaviour and values of viscosity. High and low values of $\alpha$ could result in unrealistic viscosity values.

3) Effects of the parameters on velocity at the interface are as follows:

i) Increasing $\vartheta$, all other parameters fixed, increases the velocity and vorticity, but decreases shear stress at the interface.

ii) Increasing $p_{0}$ results in a decrease in the interfacial velocity, and a decrease in the vorticity. The shear stress at the interface remains unaffected by changes in $p_{0}$.

iii) Increasing $\varepsilon$, results in an increase in the interfacial velocity, and a corresponding increase in the vorticity, and a numerical decrease in the shear stress at the interface.

iv) Increasing $\alpha$ results in a decrease in the interfacial velocity and a decrease in the vorticity, and a numerical increase in shear stress.

v) Velocity at the interface increases with increasing $D a_{1}$ for a fixed value of $D a_{2}$. Vorticity decreases, numerically, with decreasing $D a_{1}$, and shear stress at the interface increases, numerically with decreasing $D a_{1}$.

\section{References:}

[1] Allan, F.M. and Hamdan, M.H., Fluid mechanics of the interface region between porous layers, J.
Applied Math and Computation, Vol. 128, 2002, pp. $37-43$.

[2] Ford, R.A. and Hamdan, M.H., Coupled parallel flow through composite porous layers, J. Applied Math and Computation, Vol. 97, 1998, pp. 261-271.

[3] Vafai, K. and R. Thiyagaraja, Analysis of flow and heat transfer at the interface region of a porous medium. Int. J. Heat and Mass Transfer, Vol. 30(7), 1987, pp. 1391-1405.

[4] Vergne, P.H., Pressure viscosity behavior of various fluids, High Press. Res., Vol. 8, 1991, pp. 451-454.

[5] Szeri, A.Z., Fluid Film Lubrication: Theory and Design, Cambridge University Press, 1998.

[6] Martinez-Boza, F.J., Martin-Alfonso, M.J., Callegos, C. and Fernandez, M., High-pressure behavior of intermediate fuel oils, Energy Fuels, Vol. 25, 2011, pp. 138-5144.

[7] Bridgman, P.W., The Physics of High Pressure, MacMillan, New York, 1931.

[8] Bair, S. and Kottke, P., Pressure-Viscosity relationship for elstohydrodynamics. Tribology Trans. Vol 46, 2003, pp. 289-295.

[9] Savatorova, V.L. and Rajagopal, K.R., Homogenization of a generalization of Brinkman's equation for the flow of a fluid with pressure dependent viscosity through a rigid porous solid, ZAMM, Vol. 91(8), 2011, pp. 630-648.

[10] Abu Zaytoon, M.S., Jayyousi-Dajani, S. and Hamdan, M.H., Effects of the porous microstructure on the drag coefficient in flow of a fluid with pressure-dependent viscosity, International Journal of Mechanics, Vol. 15, 2021, pp. 136-144.

[11] Abu Zaytoon, M.S., Allan, F.M., Alderson, T.L. and Hamdan, M.H., Averaged equations of flow of fluid with pressure-dependent viscosity through porous media, Elixir Appl. Math. Vol. 96, 2016, pp. 41336-41340.

[12] Alharbi, S.O., Alderson, T.L. and Hamdan, M.H., Flow of a fluid with pressure-dependent viscosity through porous media, Advances in Theoretical and Appl. Mechanics, Vol. 9(1), 2016, pp. 1-9.

[13] Kannan, K. and Rajagopal, K.R., Flow through porous media due to high pressure gradients, 
Applied Mathematics and Computation, Vol. 199, 2008, pp. 748-759.

[14] Rajagopal, K.R., Saccomandi, G. and Vergori, L., Flow of fluids with pressure- and shear-dependent viscosity down an inclined plane, Journal of Fluid Mechanics, Vol. 706, 2012, pp. 173-189.

[15] Singh, A.K., Sharma, P.K. and Singh, N.P., Free convection flow with variable viscosity through horizontal channel embedded in porous medium, The Open Applied Physics Journal, Vol. 2, 2009, pp. 1119.

[16] Abu Zaytoon, M.S. and Hamdan, M.H., Fluid Mechanics at the Interface between a variable viscosity fluid layer and a variable permeability porous medium, WSEAS Transactions on Heat and Mass Transfer, Vol. 16, 2021, pp. 159-169.

[17] Nakshatrala, K.B. and Rajagopal, K.R., A numerical study of fluids with pressure-dependent viscosity flowing through a rigid porous medium, Int. J. Numer. Meth. Fluids, Vol. 67, 2011, pp. 342-368.

[18] Stokes, G.G., On the theories of the internal friction of fluids in motion, and of the equilibrium and motion of elastic solids, Trans. Camb. Philos. Soc., Vol. 8, 1845, pp. 287-305.

[19] Barus, C.J., Note on dependence of viscosity on pressure and temperature, Proceedings of the American Academy, Vol. 27, 1891, pp. 13-19.

[20] Barus, C.J., Isothermals, isopiestics and isometrics relative to viscosity, American Journal of Science, Vol. 45, 1893, pp. 87-96.

[21] Málek, J. and Rajagopal, K.R., Mathematical properties of the solutions to the equations governing the flow of fluids with pressure and shear rate dependent viscosities, in: Handbook of Mathematical Fluid Dynamics, Elsevier, 2007.

[22] Housiadas, K.D., Georgiou, G.C. and Tanner, R.I., A note on the unbounded creeping flow past a sphere for Newtonian fluids with pressure-dependent viscosity, International Journal of Engineering Science, Vol. 86, 2015, pp. 1-9.

[23] Málek, J., Necas, J. and Rajagopal, K.R., Global existence of solutions for flows of fluids with pressure and shear dependent viscosities. Applied Mathematics Letters, Vol. 15, 2002, pp. 961-967.

[24] Subramanian, S.C. and Rajagopal, K.R., A note on the flow through porous solids at high pressures, Computers and Mathematics with Applications, Vol. 53, 2007, pp. 260-275.
[25] Fusi, L., Farina, A. and Rosso, F., Mathematical models for fluids with pressure-dependent viscosity flowing in porous media, International Journal of Engineering Science, Vol. 87, 2015, pp. 110-118.

[26] Housiadas, K.D. and Georgiou, G.C., New analytical solutions for weakly compressible Newtonian Poiseuille flows with pressure-dependent viscosity, International Journal of Engineering Science, Vol. 107, 2016, pp. 13-27.

[27] Alzahrani, S.M., I. Gadoura' I. and Hamdan, M.H., A note on the flow of a fluid with pressuredependent viscosity through a porous medium with variable permeability, Journal of Modern Technology and Engineering, Vol. 2 (1), 2017, pp. 21-33.

[28] Alzahrani, S.M., I. Gadoura'I. and Hamdan, M.H., Flow down an inclined plane of a fluid with pressure-dependent viscosity through a porous medium with constant permeability, Journal of Modern Technology and Engineering, Vol. 2(2), 2017, pp. 155-166.

[29] Pozrikidis, C., Multifilm flow down an inclined plane: Simulations based on the lubrication approximation and normal-mode decomposition of linear waves. In Fluid Dynamics at Interfaces, 112128, Wei Shyy and Ranga Narayanan eds. Cambridge University Press, 1999.

\section{Contribution of individual authors}

Both authors participated in pertinent literature review.

Both authors independently obtained the solutions to governing equations.

M.S. outlined the steps to take in conducting this research.

M.S. provided calculations and graphing of results using Maple, and provided initial analysis of results. M.H. identified what quantities to calculate and values of parameters to be used.

M.H. analysed the results wrote the manuscript.

\section{Sources of funding}

This research did not receive any specific grant from funding agencies in the public, commercial, or not-for-profit sectors.

\section{Creative Commons Attribution License 4.0}

(Attribution 4.0 International, CC BY 4.0)

This article is published under the terms of the Creative Commons Attribution License 4.0 https://creativecommons.org/licenses/by/4.0/deed.en US 